\title{
Implementing an Structural Prototype of Emergency Services in Web - Application
}

\author{
Sandip Kumar Nayak ${ }^{1}$, Agraj Dutta ${ }^{2}$, Sayantan Bhowmick ${ }^{3}$, Santanu Ghosh ${ }^{4}$, Suraj Kumar Jha ${ }^{5}$, Dr. \\ Rajesh Bose $^{6}$, Dr. Sandip Roy ${ }^{7}$ \\ 1, 2, 3, 4, 5, 6, 7 Department of Computational Science, Brainware University
}

\begin{abstract}
Emergency services are highly needed for all in nowadays. Quick emergency services can save someone's life. Urgency never comes with a prior indication. In real-world scenarios, getting such services and reporting is a real challenge. Emergency services are government agencies whose job it is to respond quickly to emergencies when they arise, including firefighters, police, medical services, blood bank service and ambulance service. These all are very essential and life-protected services for all people in society because without these services we can't survive. We know that getting quick blood services, fire department etc. are very tough for us and we need these services which ensure urgent stabilization of the situation and transport to definitive care. In our busy life, for getting quick services we've made a software and for this every people will get all those essential services very easily and too fast. In this project, we have introduced a web application which provides the information of all emergency services and which can be accessed by all users 24 hours a day, 7 days a week. Some features will be available like finding nearby blood bank, medical services in whole night and from the police station to the fire department all in one place. Nowadays for this pandemic situation nobody knows when the problem will create. By this web-application everybody will get to know the all information from medical services to police station. Urgency never comes with a prior indication. We know that in real-world scenarios, survive is a big challenge for all and detecting such emergencies and reporting them is a real challenge. For society we want to provide quick emergency services and that's why we've made it. So, we think this application will help all people.
\end{abstract}

Keywords: Emergency services, web application, intelligent detection of heartrate, blood bank, fire department, medical services, police station.

\section{Introduction}

Any emergencies situation can happen anytime without any prediction. For crime, accidents, health problems, emergency services are needed urgently. The emergency service of any agencies are big responsibility that how to reach the emergency destination as soon as possible for saving lives without any loss. Government agencies and private organizations are providing the all facilities for getting emergency services. [1].

In this world maximum people are living in urban areas and a large number of people use the smartphones. Nowadays smartphone is the fast communication system in the world and computing media for people's daily tasks. All people are getting updated by the use of social media and participates the all social event by social media. [2,17,18,19]. For example, in July 2012, during the Beijing floods, people used Twitter to provide information on flooded areas and associatively developed a live crisis map of the impact of the floods using Google Maps.

Emergencies can make a person helpless but do not refer to disaster all time. Even little disruptive events can be called as emergencies but that are also an emergency and some time it makes big issue so that time need a quick and coordinated responses [3]. Emergency response activities are carried out during the period that begins with the detection of an emergency event and ends with the stabilization of the situation [4]. Traditionally, when emergencies occur you can quickly report emergencies through codes and access emergency services [5]. Different countries or states have their different codes in order to improve communication with the answering service and enable an immediate answer. To use it, a person needs a telephone only to call the emergency number of the location where the emergency needed. Some places have poor network service problems so for them the telephone line service available. A common problem happens with using phone calls to providing help at the correct location of the caller. When an emergency occurs, the nature of the emergency and its location are critical to any dispatcher. Unfortunately, getting the correct location information can become problematic when the caller does not know their exact location or the dispatcher has difficulty getting the correct location and information from the caller. 
Therefore, for developing countries this document is helping the country and develop a web application for getting emergency services quickly. The rest of this article is structured as follows: Section 2 presents related work. Section 3 presents the design of the proposed system. Section 4 analyzes the implementation of the proposed system as a mobile application. Section 5 discusses the results obtained and Section 6 concludes the article.

\section{Related Work}

In paper [6] the authors provides a beautiful mobile app that is related with the disaster environment called HelpX. It's a new approach to using Wi-Fi to enable smartphones for communicate during the time of disaster. Without any network of smartphone service providers this app works and the message forwards very intelligently by the using of Forward algorithms. After restored the services, everything will be recorded that the emergency case happens and this all will be stored in central HelpX server. Even the missing person will be found out by this information from the cell phone.

GreatCall [7] provides us easy use of friendly wireless services. This wireless system has a one-button device but it's very easy to use and like a normal mobile phone app. The most useful service is five stars ( 5 stars), by this services a person can contact directly with the service assistant for emergency case by pressing of help button. The main feature of this service is quick response with a perfect agent. It's a highly recommended personal device that is primarily used in USA, most of the citizens stay home lonely and someone notices another one for any emergency cases that is very important.

In an article [8] authors offers another good application. FAD is provided some instructions to include for primarily care of patients from the Android smartphone. For finding nearest hospital, the navigation features with the Google Maps API has been added. For getting the shortest route this navigation features help the patient to find out the hospital.

In all over America, the widely used mobile app is very helpful for all. The system is easy to use for all and it's available in both web and app format. A person can upload his own photo that will be automatically uploaded in the emergency site and by this the app users gets alert about emergency situation with the proper location and photo. So, for direct response a person can help others by this app in the emergency situation. [9]. Emergency alerts featured: There is two-way communication system included: Once the user receives an alert message on his / her phone, he / she can also reply with the massage and can add any other details information about the emergency situation. Here rescue teams and many other assistants are available and they always help user to the right directions and get out of such a situation. Reports: Every smart phone user has to install the app on their cell phone and then he/she can send a first report to others which should include text and pictures. This is the starting point of the app as the user certifies an emergency and sends over. Photos sharing features: this is a very great feature in this app to Share live photos with a security person or any other nearby user by which the helping authority can understand the actual problem. GPS\& Mapping: just like Google maps here you can also select your current location; the app locates the emergency location and advises others on the issue with location details.

Fajardo, et.al (2010) developed "A Mobile Disaster Management System Using the Android Technology" or simply called My Disaster Droid to explore the optimal path along towards geographic locations that volunteers and rescuers must take to serve the maximum number of people and provide more and more coverage of the area within a minimum time.[10]

"A Web-Based Incident Reporting System and Multidisciplinary Collaborative Projects for Patient Safety in a Japanese Hospital" developed by K Nakajima, et. al (2016) which is currently recognized as a very helpful tool for patient safety in individual hospitals as well as at the national level. Using a computerized system in minimum settings such as intensive care units was successful in involving physicians more in the reports, where they accounted for more than $20 \%$ of the total number of reports. Professional groups taking action about the incident report, including making recommendations on restrictions of high-risk drug storage areas and the establishment of the Clinical Engineering Department of the Central Administration of Medical Devices; In addition to reporting incidents and a faster response to issues revealed as a result of such reporting - which were the barriers we encountered in delivering patient safety programs, they could be resolved through a web-based incident reporting system that simplifies the process of reporting and information sharing. [11]

The Life Bank App is an intuitively blood giver database that moves committed Africans to grant blood and spare lives in their bunch [12]. It may be a wander commercial center for blood gift. The application looks for the accessible blood and blood things that patients require in its database. With a dexterous coordination system set up, things are conveyed to patients on time. An extension of this application, the LifeBankPlus may be a shrewd blood framework that interfaces clinics and blood banks and makes a difference people, discover blood required speedier, more secure, and cheaper. Be that as it may, when a client orders for blood the framework does not give the closest center to where the specific blood sort can be gotten, it as it were gives a few areas without sorting them on the premise of vicinity. The Area Based Online Blood Bank Framework (LOBB) employments a Worldwide Situating Framework and closest neighbour calculation for essential blood transfusion administrations [13]. The most point is to supply a quick and efficient way to pick up consideration of potential benefactors within the require of hour. It incorporates the utilize of SMS and E-mail administrations such that the benefactors can find the patient when the ask for blood is created. The framework makes a difference within the handle of blood gift. It comprises of a web application which acts as an interface for the clients of the framework and a database for putting away the donor's information, blood bank subtle elements and clinic points of interest. The framework was built to find as it were benefactors, and not blood bank centers the central blood bank database with against GPS versatile framework was basically planned to have a central database of blood benefactors and make a 
clinic organization to find the show range of a benefactor within the occasion of emergencies or crises. It utilizes GPS with extraordinary precision to find the position of any of the closest benefactors through a web door [14]. The clients of the frameworks are directors (this might be a common body from the central blood bank agency) blood banks, healing centers and clinics. Lay people don't have get to the framework. The Optimal facility for following the area of blood bank and benefactor may be a proposed framework which is pointed at tackling existing issues with other framework utilizing Worldwide Situating Framework (GPS) for following of nearest blood banks and givers. It requires an android gadget that underpins a GPS. The application at that point has got to be introduced on the gadget. Amid the pressing require of blood, a client can immediately get to individuals with a specific, or related blood gather and contact them through phone calls or content messages through the application [15]. The Android blood benefactor lifesaving application on the other hand may be a web application that interfacing all benefactors and patients. The framework permits people to enrol as benefactors, and they can get an SMS from neighbourhood clients who require blood to donate in occurrences of require [16]. The framework be that as it may, does not supply the genuine time area of the giver, as his enrolled address might contrast from his current area. The survey appears a few existing blood bank administration framework actualized but a certain number of them are not display inside the geological area of Nigeria. The as it were show one is the LifeBank app which as it were offers area on where blood can be gotten, be that as it may, this framework points to supply a client with the closest area of a blood bank or giver in cases of crisis.

\section{COMPARISON IN TERMS OF OTHER PORTALS: -}

In below table we are showing the application comparison with some currently used application.

\section{HelpX Portal}

1. In our portal we are providing four major services like Medicinal, Doctor, Blood Donation, and Ambulance Services at one place.

2. As we developed our web application using some advance technologies, hence we are able to provide a faster and smoother user experience.

3. In our portal, few customer executives are always remain active for proving any kind of solution to problem faced by users.

4. No other user can grant access to others account by his/her respective credentials.

5. The UI which is designed by us is so user friendly, is can used by any aged user.

6. We are concentrating on future feature updates on our portal for providing different facilities to users.

\section{Other's Portal}

1. Where as in others portal they are not providing these services altogether.

2. Some of them are still lack behind in terms of technologies, resulting in poor service and not such a great experience.

3. These portals are not so active in providing customer executive service regarding any problem faced by the users.

4. There are no such layer of securities available.

5. No such user friendly UI is available.

6. Not so regular in providing feature updates to their portals.

\section{Table-1: A comparison study}

\section{Proposed Work:}

In our proposed work, we introduce a web application that will be helpful from anywhere at any time. A remote HelpX server that's in our work, it can guide and provide the actual information for the needs of users after that users mention in the server then this message is forwarded to the server's assistant and that's why they have to take major action immediately. The time of any emergency situation, we just open our apps and select the emergency field then save it to HelpX. Now this message go to HelpX server and read the problem for analyzing then passes the message to the service providers to take the appropriate action . Mainly it's a collection of all emergency services. A single server that can solve the all emergency issues at a time.

For getting emergency services, the victim has to select the available emergency type that want to use. This apps will help the person who faces the emergency cases by the selected emergency services, to get the nearest hospital from accident place(current location of the person) the victim has to select the emergency field in apps, once provide all information to the apps after that all 
information will be saved to the HelpX server, the emergency field will provide the correct information to the person in very short time and the nearest hospital or emergency services will be displayed from the current location. This apps will also be helpful for new person who does not know the nearest hospital or healthcare in emergency and can get the proper information from this apps that will be very helpful.

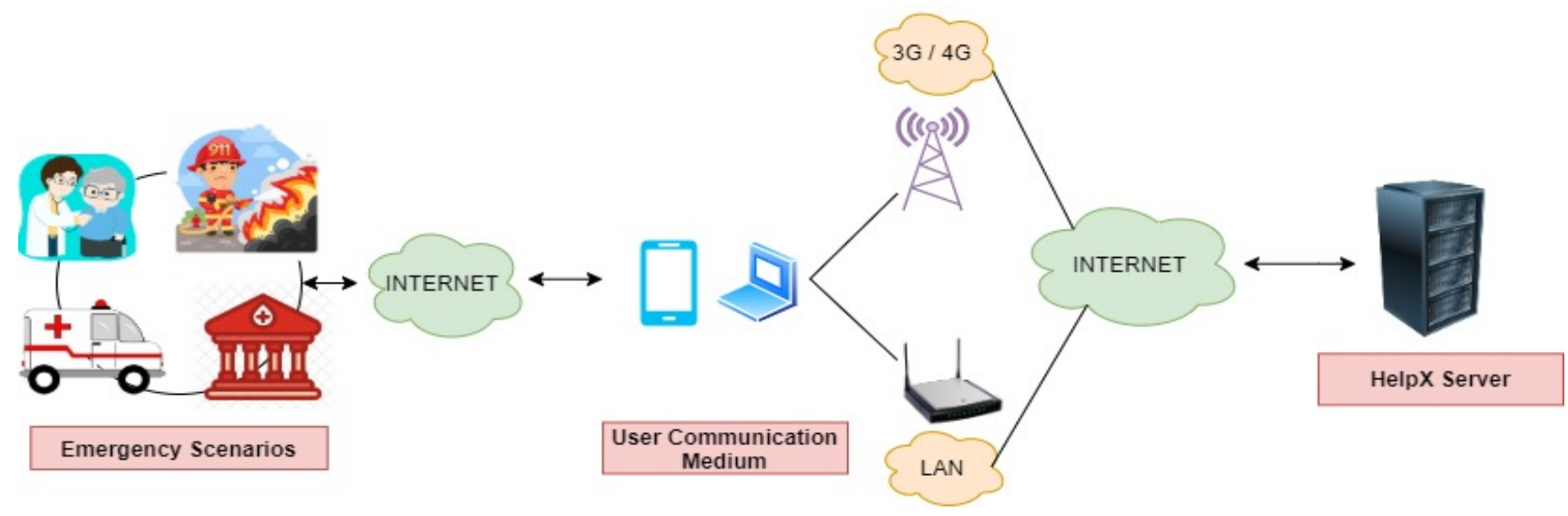

Figure 1: Proposed system Architecture Diagram

In our proposed architecture Figure1, we can see that if someone faces the emergency problem he can use this proposed web application to get the emergency services and keep the situation under control. In the time of emergency cases, if the victim will select the emergency service from the menu provided through the Wi-Fi or internet using any device like mobile or pc that will connect through LAN's to establish a proper connection to our server. After provided the details of victim, the emergency request will be sent automatically to the service providers for provide the immediate action.

A sequence diagram is used to show how operations interconnect and operate with one another and the order in which operations occur in Fig. 2.

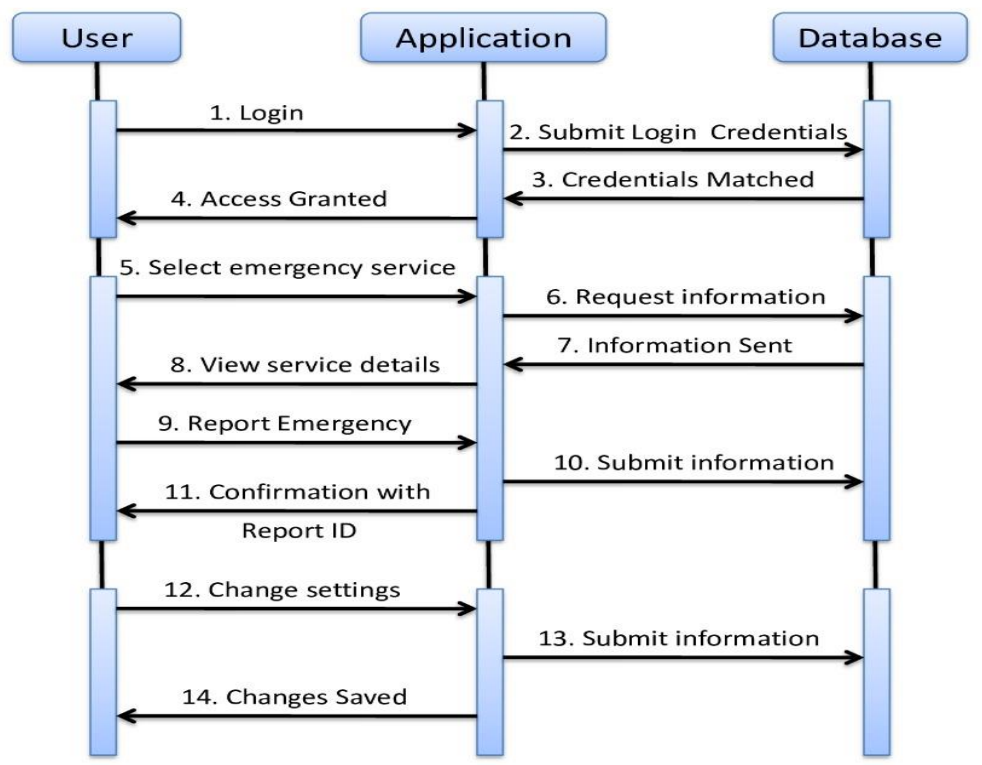

Figure 2: Proposed Sequence diagram

In Figure 2, from this diagram we can know about usage of this web application. At first, the user has to log in for using this application. Then the user has to enter his/her specific credentials into the system and if the user exist in this database the system confirms it. After confirmed the credentials, the user can use available emergency service. For getting this application's facilities, the person has to select the service then with service details the web application responses and submit the proper information. After submitted the form, the emergency system will provide individual report ID and generate this id for new user for further references. After this the user can see nearest hospital and provide the user for emergency specific service. Even changes option is available, the user can change his credentials after logged in to the system. 


\section{Experimental Work}

\subsection{Technologies Used:}

- PHP - The PHP software interacts with the web server, which is a computer software that distributes web pages to the rest of the globe. When you type a URL into the address bar of your web browser, you're requesting the web server at that URL to provide you an HTML file. The requested file is sent by the web server in response. PHP is an HTML-enabled server-side scripting language. It's used to manage dynamic content, databases, and session tracking, as well as to create full e-commerce websites. We used it as a server-side language in our suggested architecture to connect MySQL databases for storing credentials and information.

- HTML - HTML stands for Hyper Text Mark-up Language. It is the industry standard mark-up language for all major browsers on the market. HTML is a fundamental prerequisite for creating a website. The basic foundation of our website was built using HTML.

- CSS3 - CSS3 is used to format structured content and is used in conjunction with HTML to generate content structure. Font attributes, colors, text alignments, graphics, background images, tables, and other components are all within its control.

- Bootstrap - Bootstrap is a free, open-source HTML, CSS, and JS framework that includes templates for text, forms, buttons, navigation, and other user interface elements. To reduce the number of lines of code in our project, we used bootstrap. This significantly simplified our job and improved the responsiveness of our website across a wide range of devices.

- JQuery - JQuery is a lightweight JavaScript library that allows you to "write less, accomplish more." The goal of jQuery is to make using JavaScript on a website considerably easier. JQuery encapsulates many typical operations that require multiple lines of JavaScript code into methods that may be called with only a single line of code. JQuery also simplifies several of JavaScript's more difficult features, such as AJAX calls and DOM manipulation.

- MySQL - MySQL is a relational database management system that is free and open-source. We've been using it to manage our databases of various services that we provide through our HelpX server.

\subsection{HelpX development}

The HelpX system includes a reported emergency list box, where all complaints are recorded to the HelpX database and updated with requests. The server is responsible for keeping track of new requests as they arrive. All reported emergencies are saved with information such as the type of emergency complaint, date and time, geo-coded address of the location, latitude and longitude (spatial data), and once the emergency arrives, the emergency arrival server is responsible for determining where the emergency originated by using geo-coded address (road network address). The precise location of the emergency can also be determined via the spatial data server. After obtaining the area, the server must select the required emergency, such as medical for hospitals with ambulances, fire station facility, or police facility. The server also has the option of searching for the facility within meters, such as 500 meters, 1000 meters, 5000 meters, and so on. After you've completed all of your searches, click search to see the name of the emergency type as well as the shortest distance, which reveals the server's precise position as well as the shortest distance from the person in an emergency.

\section{Implementation of the Proposed System}

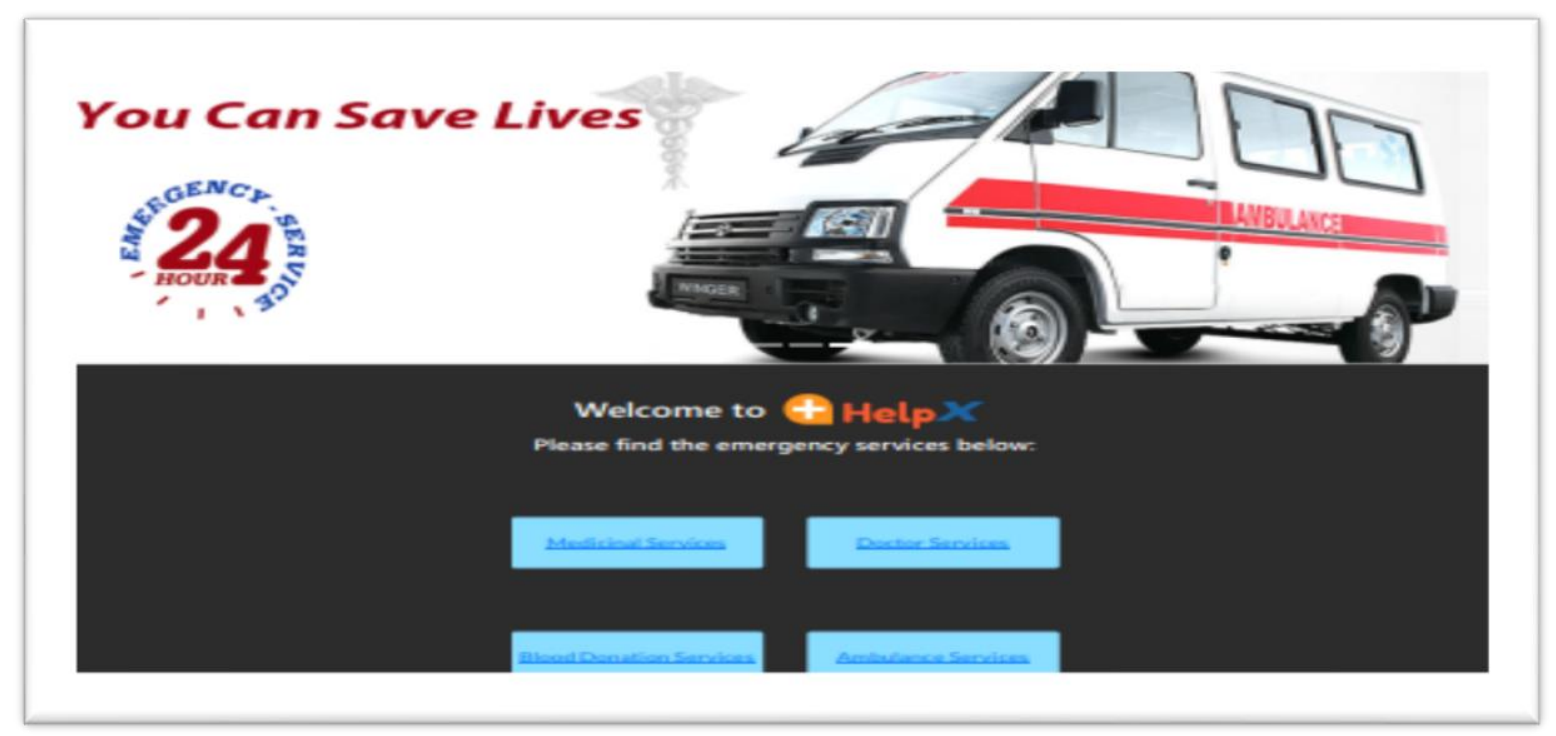

Figure 3: Proposed Architecture of Home Page 
In our proposed architecture named HelpX, which can be seen in Figure 3, that is basically our HOME page which opens 4 different emergency options to the person in trouble. Below the carousel we have added buttons to open an option to see the details of our services and the way we work. There will be options to register and login. New users will need to register first and existing users must log in to use the services.

\section{Medicinal Services}

First of all we can see at the top its showing " $24 / 7$ medicine center", by using of header font we increased the size of " $24 / 7$ medicine center". In this, some sub options are available like home, medicine, account, feedback, registration, about us etc. These all are colorful options. Just click on this option and we will watch a different colorful option. At first people have to log in in this application then they can use it. We can see a search option in this page, by this search option people can get their needed medicine. In the carousel, the picture will change randomly. People can give feedback by using feedback option. If someone faces some problem in this application, they can contact with higher authority by using Contact Us option. If someone wants to know about this medical service then they can choose About Us option.

This page opens many options to the user. From here you can go to the home page which is the main page by clicking the HOME button. Also we added sub options here. User can visit our medicine page by clicking the medicine button. New user or existing user can register or login themselves by clicking the ACCOUNT button option. Then you can also share your feedback and know more about us by clicking the FEEDBACK and ABOUT US button respectively. User can also search for their urgently needed medicine by clicking the search bar at the top right corner.

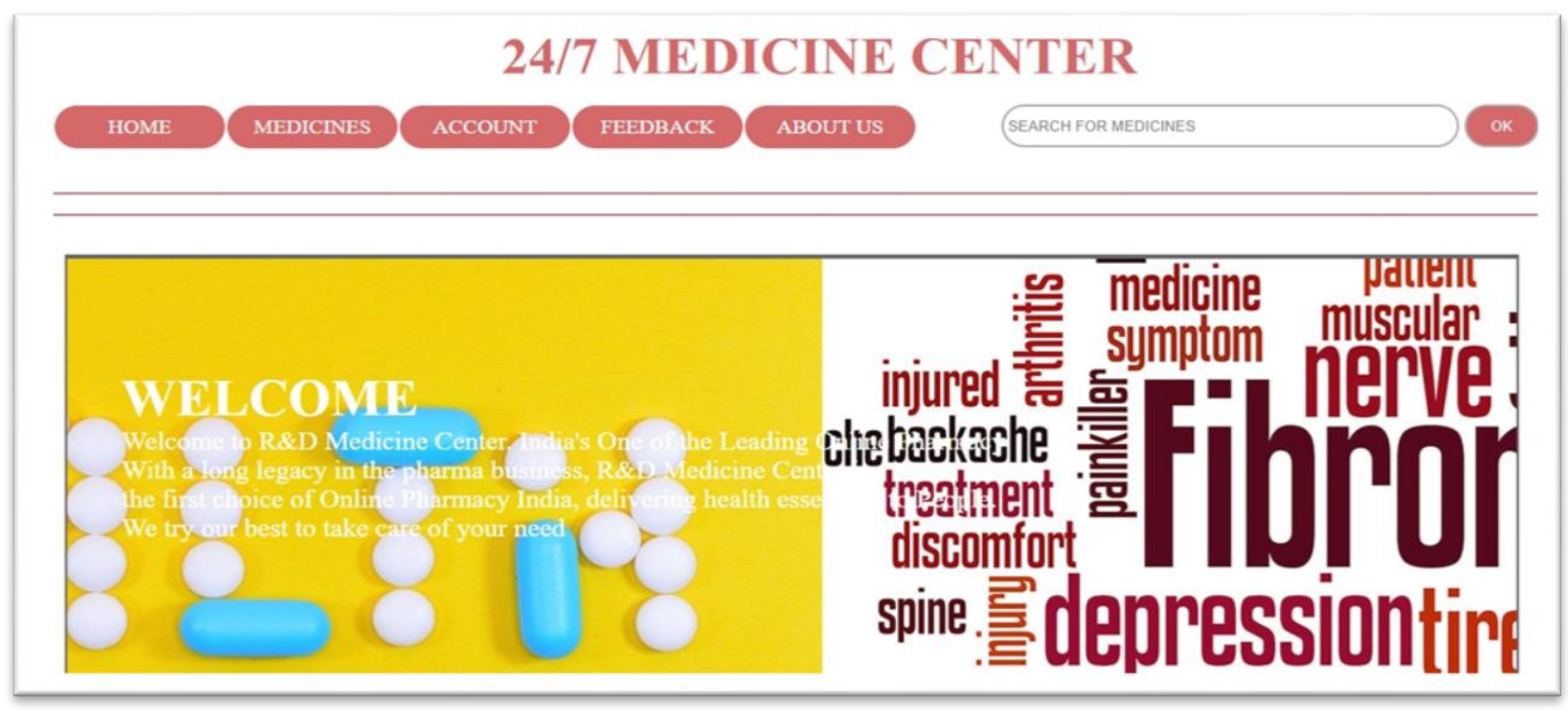

Figure 4: Home Page of Medicinal Services

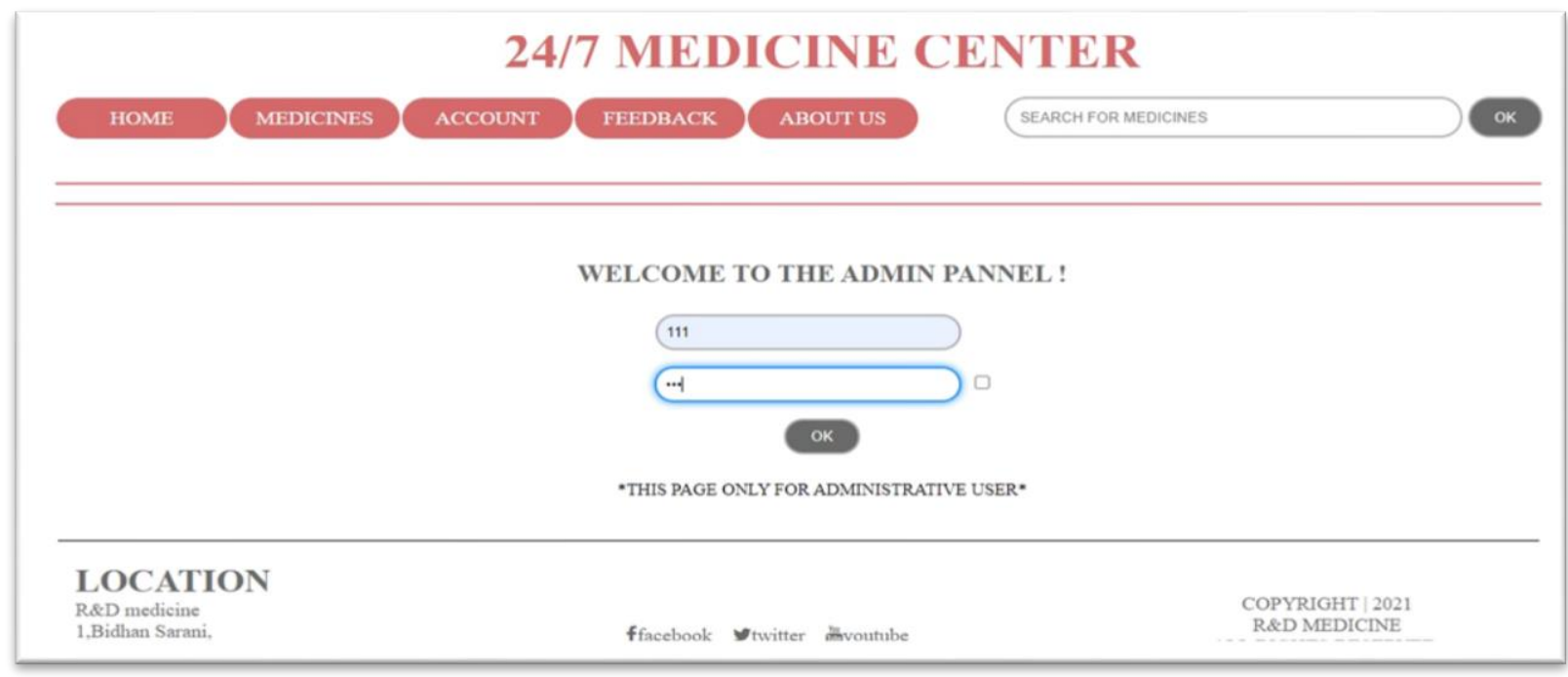

Figure 5: Admin Login Page 
We created an option admin that's in footer portion. It is only used for login. Admin can log in by own username and password, that's how admin can access the whole of admin panel. We used header and footer in this page. We used different font, color etc. for showing it amazing. Admin can check order id, transaction, how many orders has come, payment status, feedback all of this . Even admin can solve all of problems.

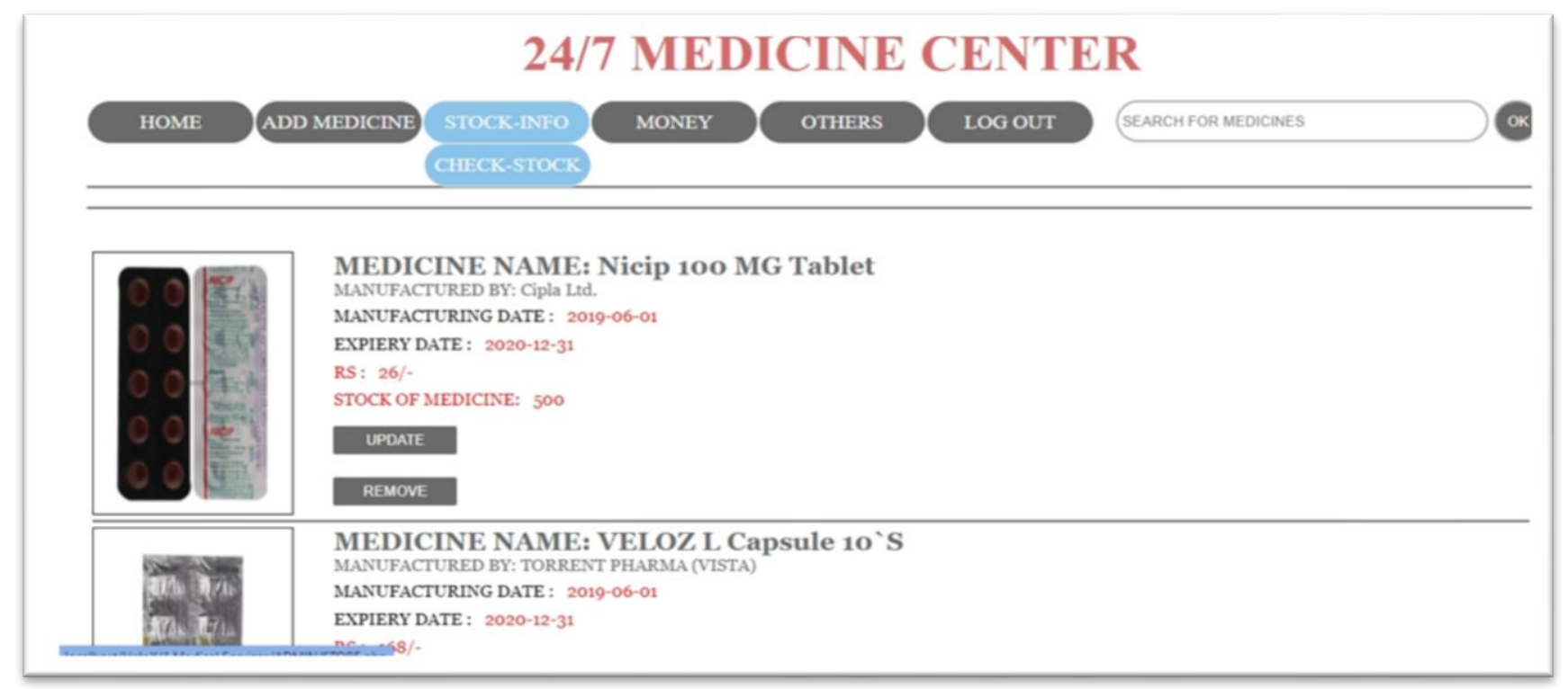

Figure 6: Check Stock Page

After logged in admin panel, admin can watch home, add medicine, check medicine, stock info option. In stock info option admin can check anything and can update details of medicine, how many medicines are available, expiry date, price, manufacturer, etc. Even admin can delete medicine from check info option and much more options are there too.

\section{Doctor Services}

This is the home page of doctor services in our web service. Without log in or register, every people can access this page without $\log$ in. From this page user can know that which specialist doctors are available like cardiologist, kidney specialist, eye specialist etc. for different kind of diseases. If the cursor will go to on the photo of different kind of sectional photo then user can see which doctors are available.

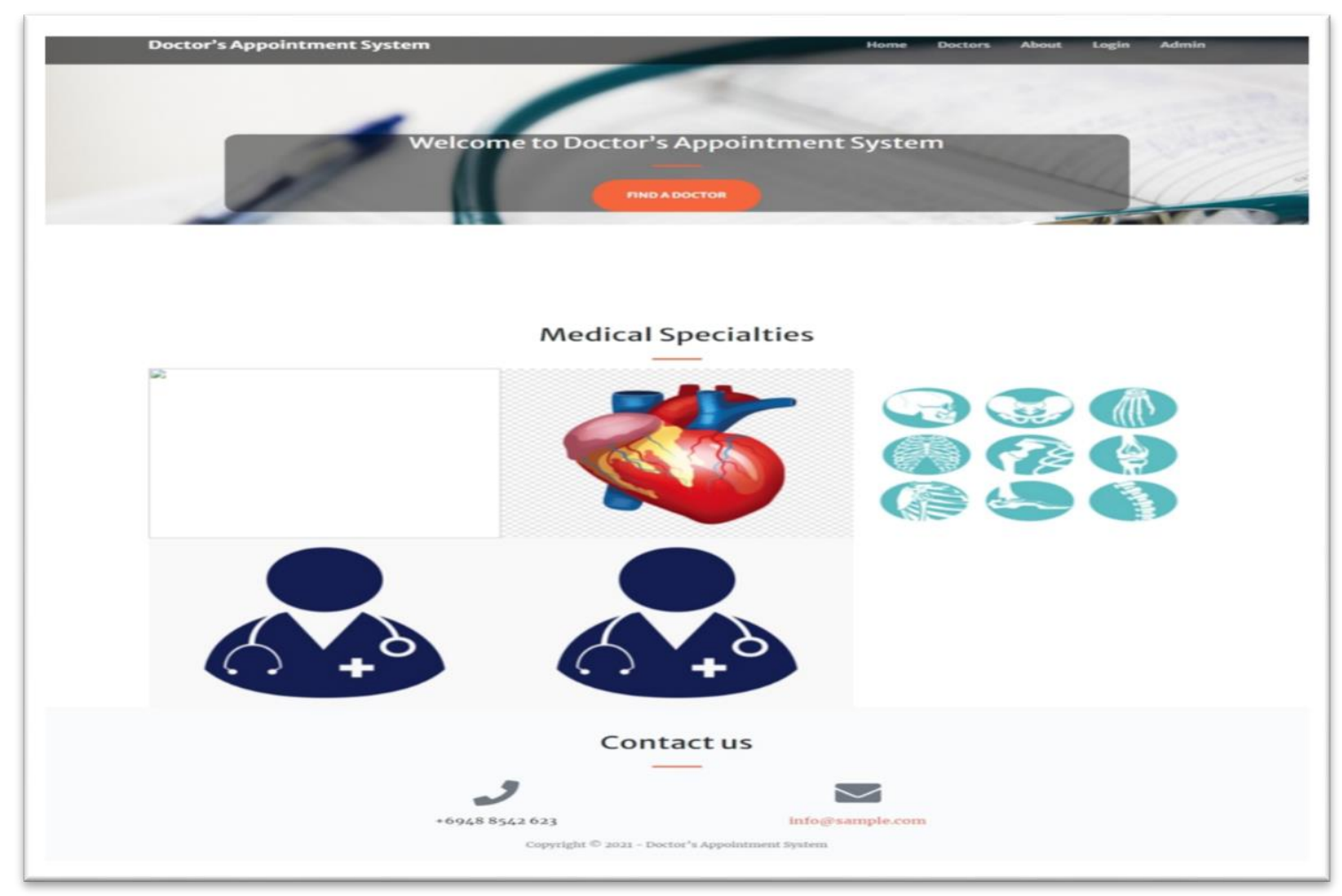

Figure 7: Doctor's Service Home Page 


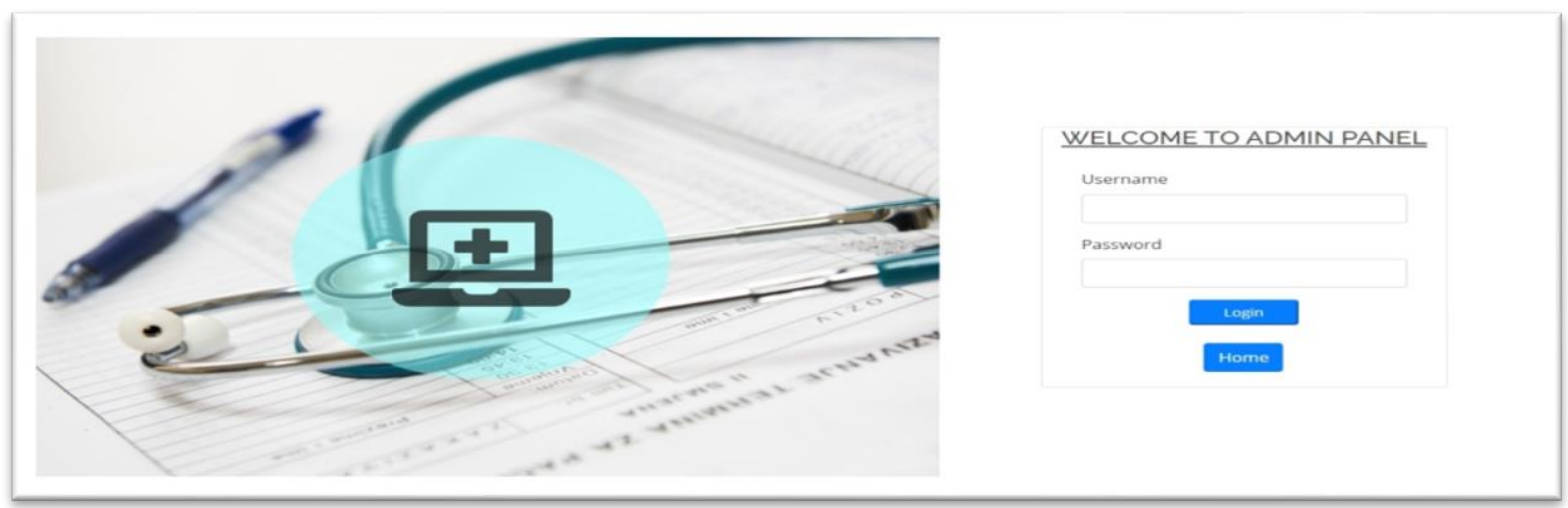

Figure 8: Admin Login Page

This is the main home page of this web service. In header, we can see a navigation bar where this admin option is presented. After clicked on this admin option, log in option will be available. In this page user can provide username and password for accessing this web service. We can see a home option, after clicked on this home option user can access this web service by their respective credentials and if an admin will click on this option then this page will be redirected to admin panel. Admin can access the admin panel after providing username and password. For creating Doctor ID and doctor's appointment, admin can create that by this admin panel and after entering in this admin panel doctor can check their appointment from this admin panel. Even an admin can see all the user id, username of users and can see doctor's appointment.

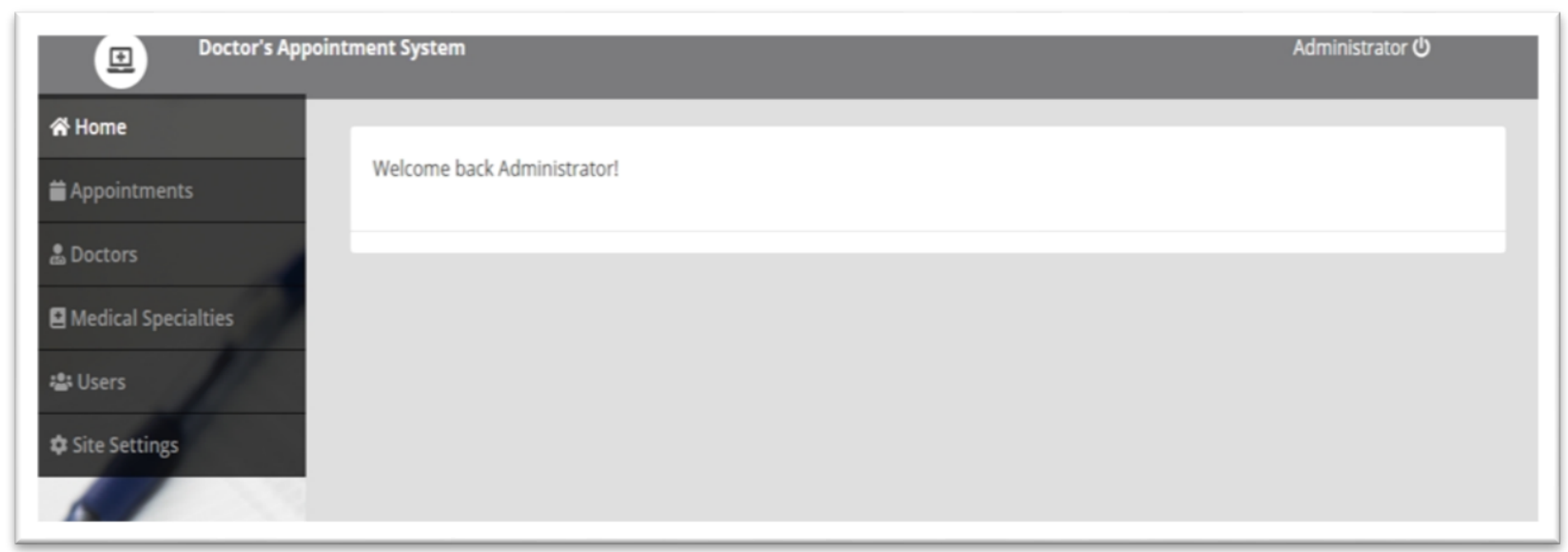

Figure 9: Home Page of Admin

When the main admin login in the admin panel with his/her own credential this page will open. Here we used a nave bar at the top where icon added at the left and doctor appointment system with a different bolt used. And top right corner we add a administrator button which can help you to logout. A simple banner said welcome administrator with a normal font. In this side admin can completely control the doctor's service.

\section{Blood donation Services}

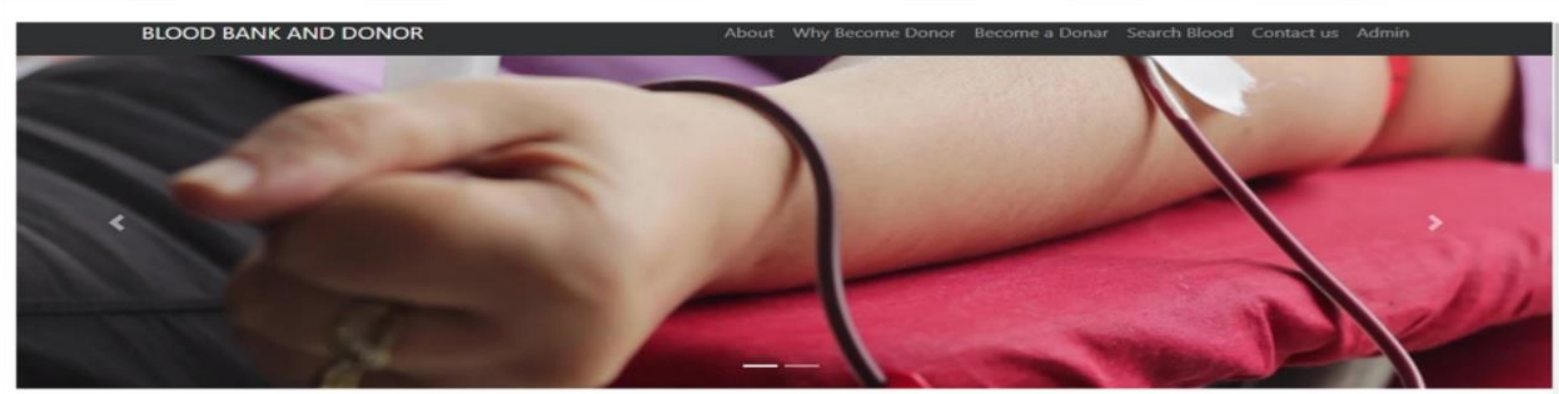

Welcome to BloodBank \& Donor

The need for blood

Blood Tips

Who you could Help

Figure 10: Home Page of Blood donation Services 
This is the home page of our blood donation service. Here we used a nave bar in header where at the top left corner you can see our service name BLOOD BANK AND DONOR and at the right side there are many options by which we are able to know about our services. And also here we used colossal by which the pictures changing one by one here. At the bottom there are many information and tips about blood donation.

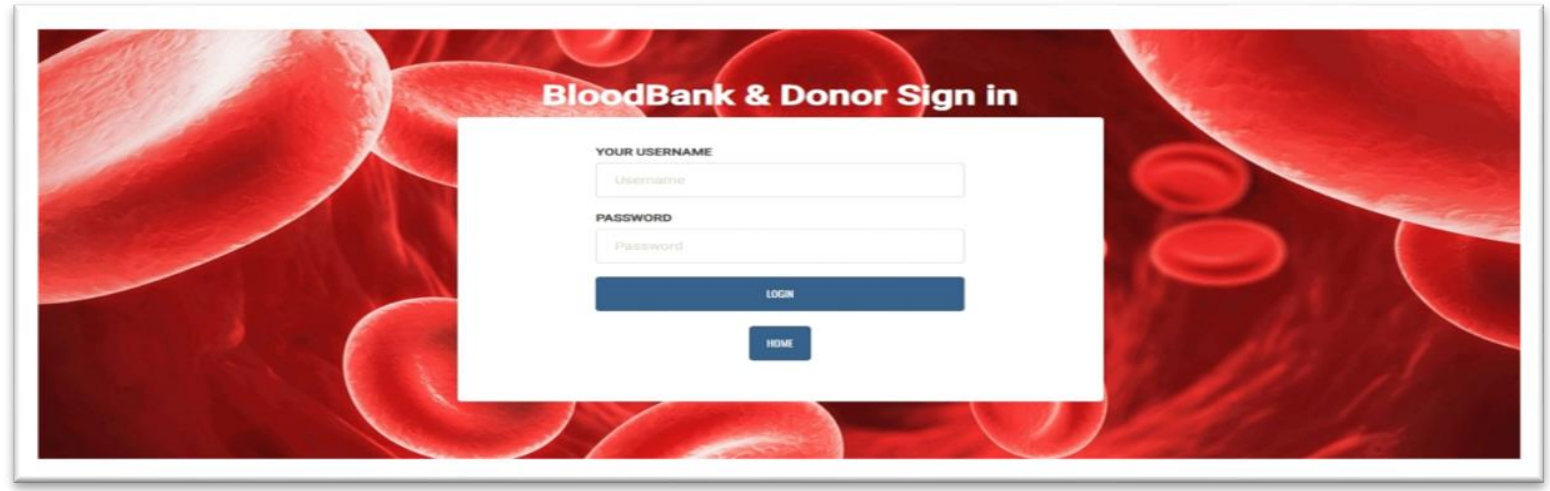

Figure 11: Admin's login Page of Blood bank Services

It's the $\log$ in page in blood service section for admin. Admin can log in by providing own username and password. By the "home" button admin can go to blood service's home page. We created "blood bank \& donor sign in" heading by using big font size and added a picture in background.

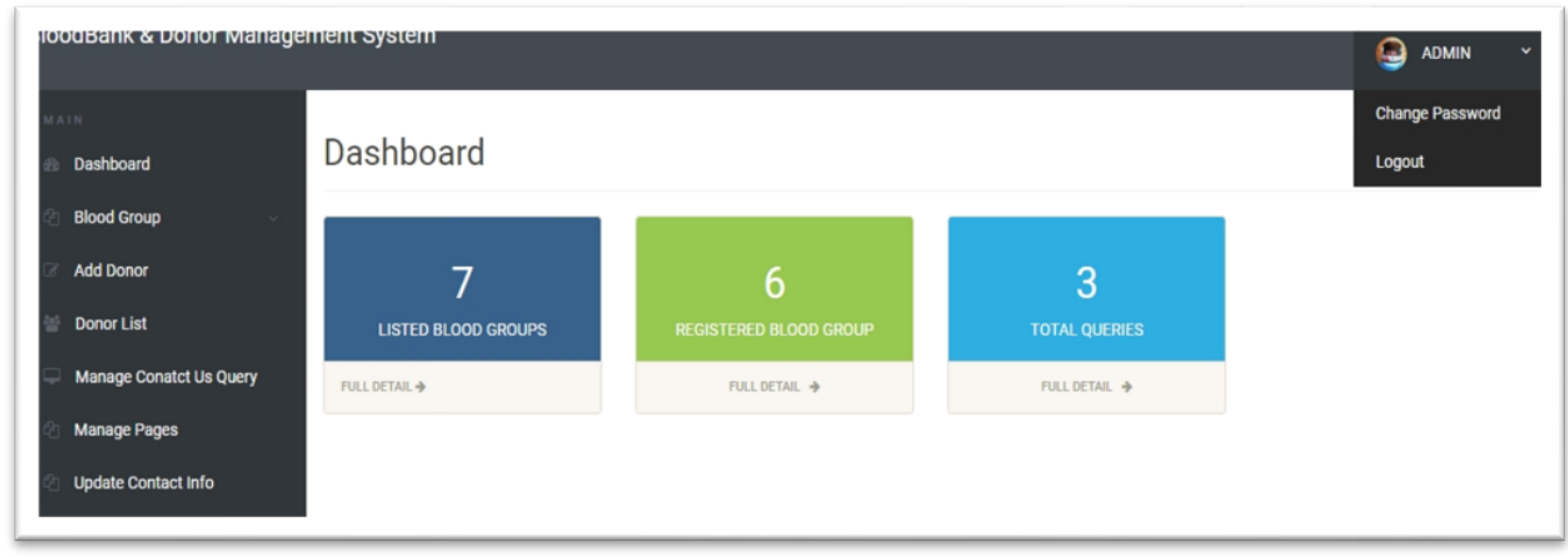

Figure 12: Dashboard of Admin Panel Page

This is the dashboard of the admin panel of our service. From here you can check many things as an admin. At the right side of the page this is the first option among the seven options. You can see the details about listed blood groups, registered blood group and total queries of our service. At the top right corner there is a dropdown button named admin by which you can change the admin panel password and directly logout from admin panel.

\section{Ambulance Services}

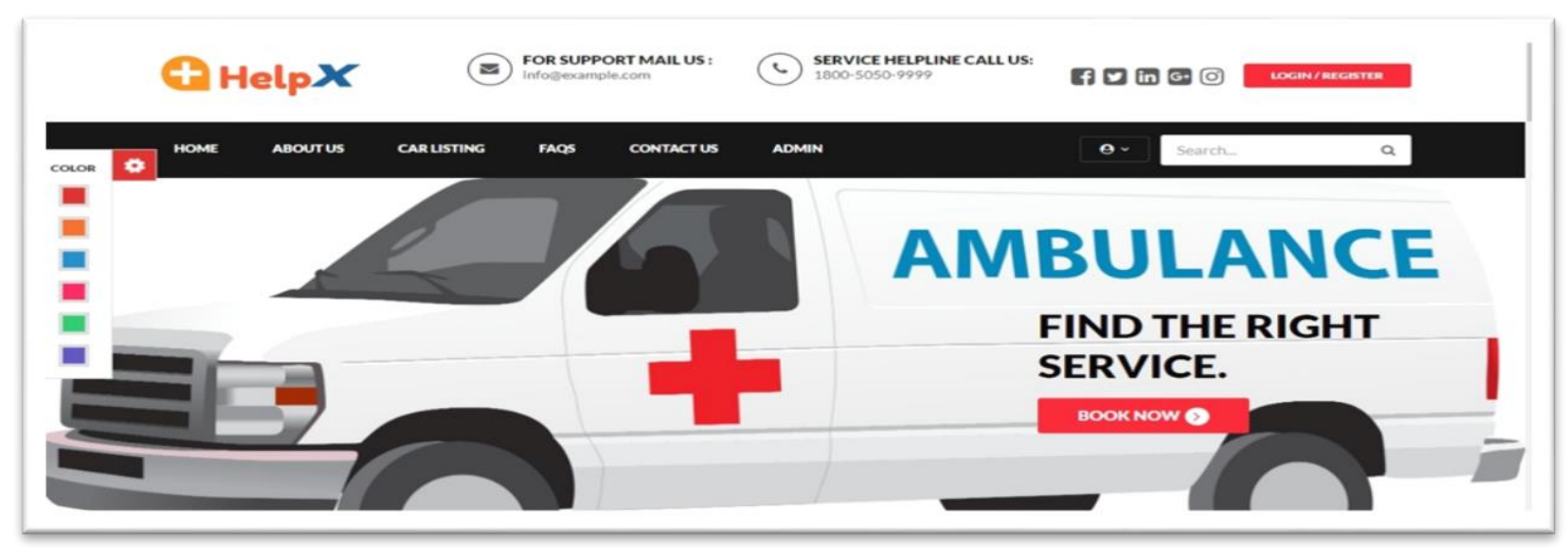

Figure 13: Home Page of Ambulance Services 
This is our home page of ambulance service. In this page we used a header with our server logo, contact support and our social media link. Also at the right side of the header there is a button for Login and Register. We used a nave bar here in which there are some buttons name HOME, ABOUT US, CARLISTING, FAQS, CONTACT US and ADMIN. There is a search bar at the right side of the nave bar and also user can change the page color through the color option at the right side. At the middle we used an image slide show for which the image is changing one by one.

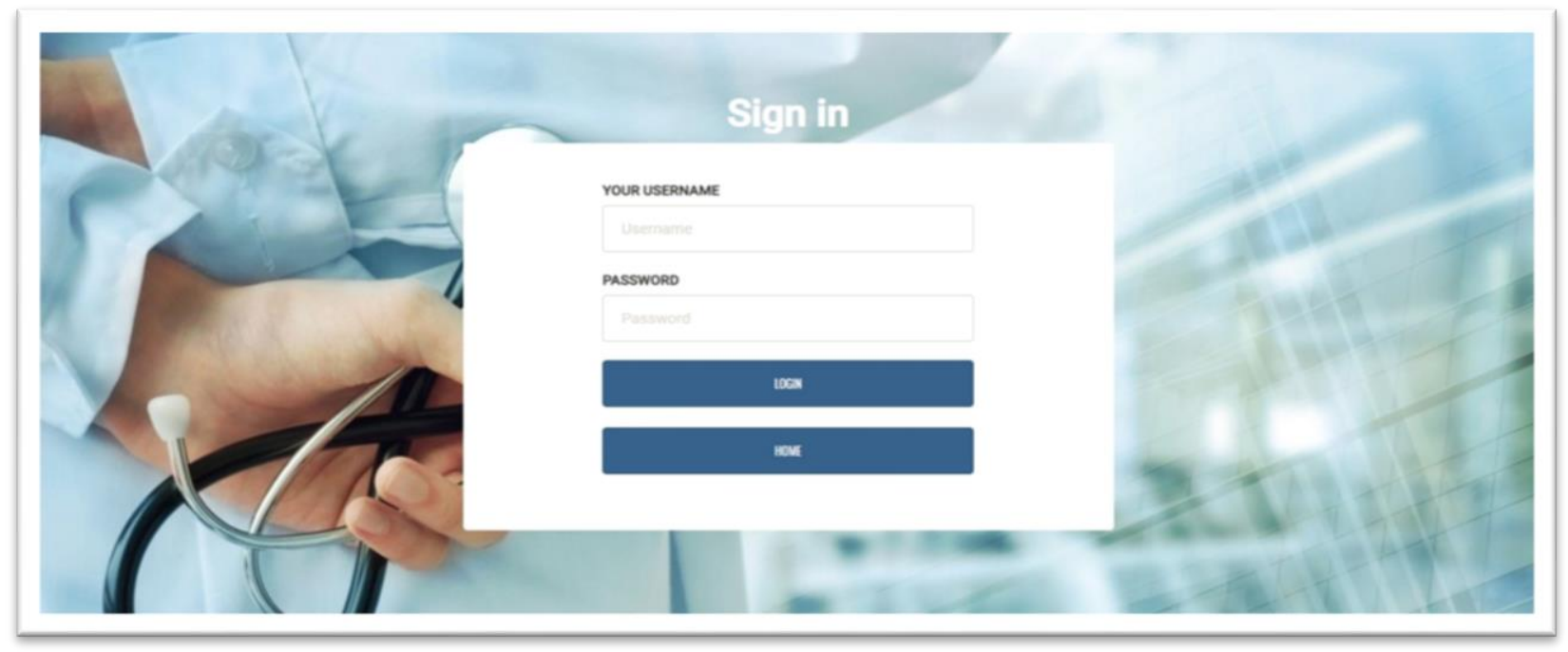

Figure 14: Admin Sign in Page

This is the admin login page of our ambulance service. User need to enter the username and password of the admin panel through which they can access the admin panel. There is a home button which directly linked to the home page of our ambulance service. Also we used a background image to make our page more attractive.

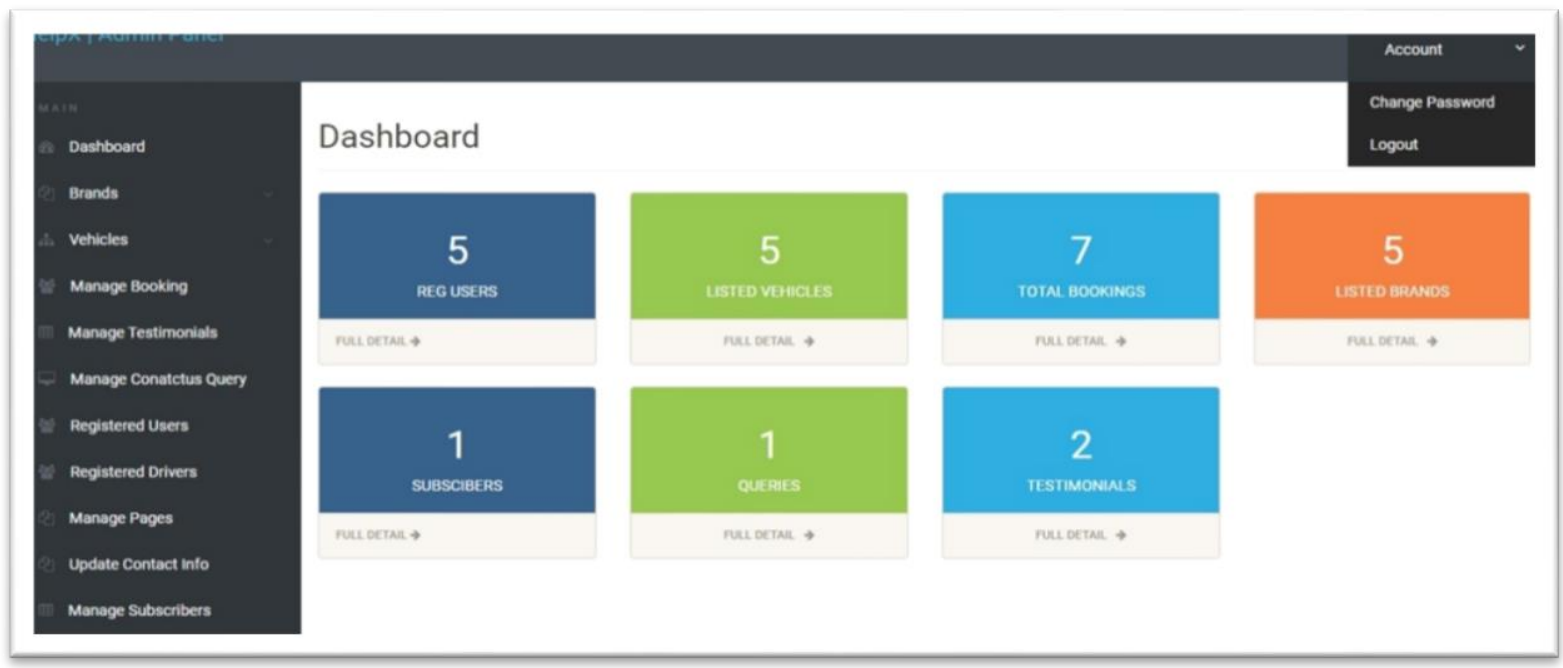

Figure 15: Dashboard of Admin panel Page

So this is basically the dashboard of the admin panel of our ambulance service. In the left side of the nave bar we added our server name and the right side there is a Account named button through which admin can change the password of admin panel and directly logout from it. In the left side there is a menu bar and at the middle admin can see all the activity performed by customer of this side like total registered users, listed vehicles, total booking etc.

\section{Conclusion}

The functionality of the Web application indicates that it can prevent a significant number of fatalities due to inadequate facilities and time. An app such as this can replace many traditional methods such as telephone calls, text messages, and emailing. In the future, it may also include many other emergency services too. All the major bugs have been removed from this application before submission, it works flawlessly in the vast majority of cases. In certain cases, the web application will not work, such as when we try to retrieve a password and the mailer service is not set up, a connection error will appear. This problem will be resolved in upcoming updates. The Web application is up and running properly. We will attempt to update it regularly with new features to 
keep it current. An architecture is described that can assist people in any emergency by providing assistance from the rescue team using android smartphones or a computer.

\section{References}

[1] "The Role of Government in a Disaster," in The Disaster Handbook 1998 National Edition, Florida, University of Florida Cooperative Extension Service - Institute of Food and Agricultural Sciences, pp. 1-6.

[2] Fuming Shih, OshaniSeneviratne, IlariaLiccardi, Evan Patton, Patrick Meier, Carlos Castillo, "Democratizing mobile app development for disaster management", AIIP '13 Joint Proceedings of the Workshop on AI Problems and Approaches for Intelligent Environments and Workshop on Semantic Cities, Pages 39-42.

[3] S. Shan, L. Wang, L. Li, and Y. Chen, An emergency response decision support system framework for application in egovernment, Information Technology Management 13 (2012), 411427

[4] M. Turoff, Past and future emergency response information systems, Communications of the ACM 45 (2002), 29-32

[5] D. Lorenzi, J. Vaidya, S. Chun, B. Shafiq, V. Naik, V. Atluri, and N. Adam, Community based emergency response. In Proceedings of the 14th Annual International Conference on Digital Government Research (2013) 82-91

[6] OsnatMokryn, DrorKarmi, AkivaElkayam, Tomer Teller "Help Me: Opportunistic Smart Rescue Application and System" The 11th Annual Mediterranean Ad Hoc Networking Workshop (MedHoc-Net), 2012.

[7] Official GreatCall Website. (2013, January). [Online]. Available: http://www.greatcall.com

[8] KomwitSurachat, SupasitKajkamhaeng, KasikritDamkliang, WatanyooTiprat, and aninnuchWacharanimit, "First Aid Application on Mobile Device", International Scholarly and Scientific Research \& Innovation 7(5) 2013,pp-361-366.

[9] Official ELERTS Website. (2013, January). [Online]. Available: http://elerts.com

[10] Eguchi, R. (2008, October). The Application of Remote Sensing Technologies for Disaster Management. Retrieved from www.iitk.ac.in/nicee/wcee/article/14_K004.pdf.

[11] YunusEmre Aydin (2006, May). Web Based Multi-Participant Spatial Data Entry in Crime Mapping”. Retrieved from https://etd.lib.metu.edu.tr/upload/12607250/index.pdf.

[12] Suchitra, R., Sannitha, M.L., Ravikanth, J., Anusha, G., Abhilash, B.: Location Based Online Blood Bank System. BEST Int. J. Manag. Inf. Technol. Eng. (BEST IJMITE). 3, 1-4 (2015).

[13] Jonathan, O., Azeta, A., Misra, S. Development of prepaid electricity payment system for a university community using the LUHN algorithm. Lecture Notes of the Institute of Computer Sciences, Social-Informatics and Telecommunications (2018), 204, $107-114$

[14] Meshram, A., Bramhe, P., Jaronde, S., Ambatker, P.: Central Blood Bank Database With Anti GPS Mobile System. 3, 970972 (2013).

[15] Pawar, R., Thigale, S., Walekar, P., Thakar, G., Joshi, P.D.: Optimal Facility for Location Tracking of Blood Bank and Donor. Int. Res. J. Eng. Technol. 3, 1325-1330 (2016).

[16] Debabrata Sarddar, Sandip Roy, and Rajesh Bose, “An Efficient Edge Servers Selection in Content Delivery Network Using Voronoi Diagram", International Journal on Recent and Innovation Trends in Computing and Communication, Vol. 2, No. 8, pp 2326-2330.

[17] Dr. Debabrata Sarddar, Raktim Kumar Dey, Dr. Rajesh Bose, Dr. Sandip Roy,“ Topic Modeling as a Tool to Gauge Political Sentiments from Twitter Feeds ”,International Journal of Natural Computing Research Volume 9 • Issue 2 • April-June 2020 .IGI .

[18] Dr. Rajesh Bose, Dr. P.S.Aithal, Dr. Sandip Roy, "Survey of Twitter Viewpoint on Application of Drugs by VADER Sentiment Analysis among Distinct Countries”, International Journal of Management, Technology, and Social Sciences (IJMTS), ISSN: 2581-6012, Vol. 6, No. 1, March 2021.

[19] Sandipan Biswas, Indranil Sarkar, Prasenjit Das, Rajesh Bose, and Sandip Roy, ".Examining the Effects of Pandemics on Stock Market Trends through Sentiment Analysis", Journal of Xidian University, Vol. 14, No. 6, June 2020.

[20] Rajesh Bose, Raktim Kumar Dey, and Sandip Roy, "Sentiment Analysis on Online Product. Information and Communication Technology for Sustainable Development, pp. 559 - 569, 2020, Springer, Singapore. 